\title{
Design and construction of a solid switchable phantom for diffuse optical imaging
}

\author{
Antonio Pifferi ${ }^{1,2}$, Fabrizio Martelli ${ }^{3}$, Lorenzo Spinelli ${ }^{2}$, Andrea Farina ${ }^{2}$, Rebecca Re $^{1}$, Giovanna \\ Quarto ${ }^{1}$, Sanathana Konugolu Venkata Sekar ${ }^{1}$, Alessandro Torricelli ${ }^{1}$, Rinaldo Cubeddu ${ }^{1}$, Mikhail \\ Mazurenka ${ }^{4,5}$, Rainer Macdonald ${ }^{5}$, Heidrun Wabnitz ${ }^{5}$ \\ ${ }^{1}$ Dipartimento di Fisica, Politecnico di Milano, Italy; ${ }^{2}$ Istituto di Fotonica e Nanotecnologie, \\ Consiglio Nazionale delle Ricerche, Milano, Italy; ${ }^{3}$ Dipartimento di Fisica e Astronomia, Università \\ degli Studi di Firenze, Sesto Fiorentino, Italy; ${ }^{4}$ Hannoversches Zentrum für Optische Technologien, \\ Hannover, Germany; ${ }^{5}$ Physikalisch-Technische Bundesanstalt (PTB), Berlin, Germany
}

\begin{abstract}
We propose a simple and reliable solid phantom for mimicking realistic localized absorption changes within a diffusive medium. The phantom is based on a solid matrix holding a movable black inclusion embedded in a rod. Translating the rod parallel to the phantom surface, the inhomogeneity can be positioned beneath the source-detector pair (perturbed case) or far from it (unperturbed case). Examples of time-resolved transmittance measurements and time-resolved reflectance scans are shown to demonstrate the properties and the versatility of the phantom.
\end{abstract}

Keywords: Phantom, diffuse optics, time-resolved spectroscopy, absorption, scattering

\section{INTRODUCTION}

The compelling need for standardization and quality assessment of diffuse optics instruments is a key requirement for the translation of the optical tools to effective clinical use [1]. This demands for the availability of calibrated and reliable phantoms. While homogeneous phantoms have reached a maturity stage with well characterized and reproducible optical properties, the construction of inhomogeneous phantoms - mimicking, for instance, a focal activation in the brain cortex, a change in muscle oxygenation [2] or a breast lesion - is challenging. Also, the construction of dynamic phantoms, with the chance to easily and quickly vary phantom parameters is a further need. We have previously demonstrated an equivalence relation stating that any realistic absorption change can be mimicked by a totally absorbing sphere of a proper volume [3]. Based on this concept, a liquid inhomogeneous phantom was proposed [4], using black PVC cylinders immersed in a tank of accurately characterised [5] Intralipid and ink solutions. Here we propose the realization of the same concept in a solid phantom holding a black inclusion embedded in a rod. Functional activations, dynamic changes or localized inhomogeneities can be produced by switching the phantom from the homogeneous reference state to the inhomogeneous perturbed one by translating the rod. This phantom can be used to implement the NEUROPT protocol for performance assessment of brain imagers [6].

\section{PHANTOM DESIGN}

The phantom is composed of a homogeneous matrix made of epoxy resin added with black toner and $\mathrm{TiO}_{2}$ particles, yielding an absorption coefficient of $0.1 \mathrm{~cm}^{-1}$ and a reduced scattering coefficient of $10 \mathrm{~cm}^{-1}$ at $690 \mathrm{~nm}$, as determined using a time-resolved system [7]. With the same matrix, 4 epoxy resin rods are cast, each of them embedding a different small PVC cylinder. These objects were chosen with proper dimension so as to mimic realistic changes in absorption properties, as described in Table 1. The equivalence relation holds true for any geometry, thus both for reflectance and transmittance, or even for use at a short source-detector distance $[8,9]$. 
Table 1. Set of black PVC inclusions

\begin{tabular}{cccc}
\hline diameter & height & volume & equivalent $\Delta \mu_{\mathbf{a}}$ \\
\hline $3 \mathrm{~mm}$ & $3 \mathrm{~mm}$ & $20 \mathrm{~mm}^{3}$ & $0.05 \mathrm{~cm}^{-1}$ \\
\hline $4 \mathrm{~mm}$ & $4 \mathrm{~mm}$ & $50 \mathrm{~mm}^{3}$ & $0.1 \mathrm{~cm}^{-1}$ \\
\hline $5 \mathrm{~mm}$ & $5 \mathrm{~mm}$ & $100 \mathrm{~mm}^{3}$ & $0.17 \mathrm{~cm}^{-1}$ \\
\hline $7 \mathrm{~mm}$ & $7 \mathrm{~mm}$ & $270 \mathrm{~mm}^{3}$ & $0.4 \mathrm{~cm}^{-1}$ \\
\hline
\end{tabular}

The cylinders embedding the black inclusions are freely interchangeable and can translate parallel to the upper phantom surface at a depth $\mathrm{z}=15 \mathrm{~mm}$ (Figure 1). The cylinder can be connected to a micrometer screw or to a linear actuator so as to yield precise and reproducible positioning of the inclusions. The source and detector fibers can be firmly positioned either on the upper surface (X-scan) or on the lateral surface (Z-scan), symmetrical with respect to the cylinder, and at a typical interfiber distance $\rho=30 \mathrm{~mm}$. Full details on the phantom design and characterization are provided in Ref. [10].

\section{RESULTS AND DISCUSSION}

Time-resolved transmittance measurements of the phantom were acquired using a time-resolved optical mammograph [11] performing a scan over the central area of the phantom covering a $4 \times 4 \mathrm{~cm}^{2}$ region at steps of $1 \mathrm{~mm}$. Figure 2 displays the time gated intensity acquired at a delay $t=1000 \mathrm{ps}$ with a gate width $\Delta t=500 \mathrm{ps}$. The rod is clearly visible in the image, accounting for some unwanted mismatch in optical properties or light-guiding or light-confinement effects at the interface. Nonetheless, the 4 embedded black inclusions (see Table 1) are all clearly detectable, with increasing contrast. If data are normalized to the unperturbed state (i.e. with the black inclusion shifted far from the fibers), then the rod perturbation is cancelled, and the images reveal only the PVC object (data not shown) [10].

To mimic the effect of a perturbation set at given position beneath the fiber the cylinder can be translated, holding the fibers either on the upper surface (X-scan) or on the lateral surface (Z-scan). The relative contrast produced by the optical inhomogeneity at a given photon time-of-flight $t$ can be calculated as $C(t)=-\ln \frac{\Delta R(t)}{R_{0}(t)}$, where $\Delta R=R-R_{0}$, while $R$ and $R_{0}$ are the perturbed and unperturbed (reference) reflectance measurements, respectively. The reference state is acquired when the inclusion is far from the fibers, so it can be obtained on the very same cylinder bearing the black object of interest. Examples of Z-scans performed using a state-of-the-art time-resolved functional oximeter [12] operated at an interfiber distance $\rho=30 \mathrm{~mm}$ are shown in Figure 3. All inclusions were detected with a depth sensitivity increasing upon increasing $t$.

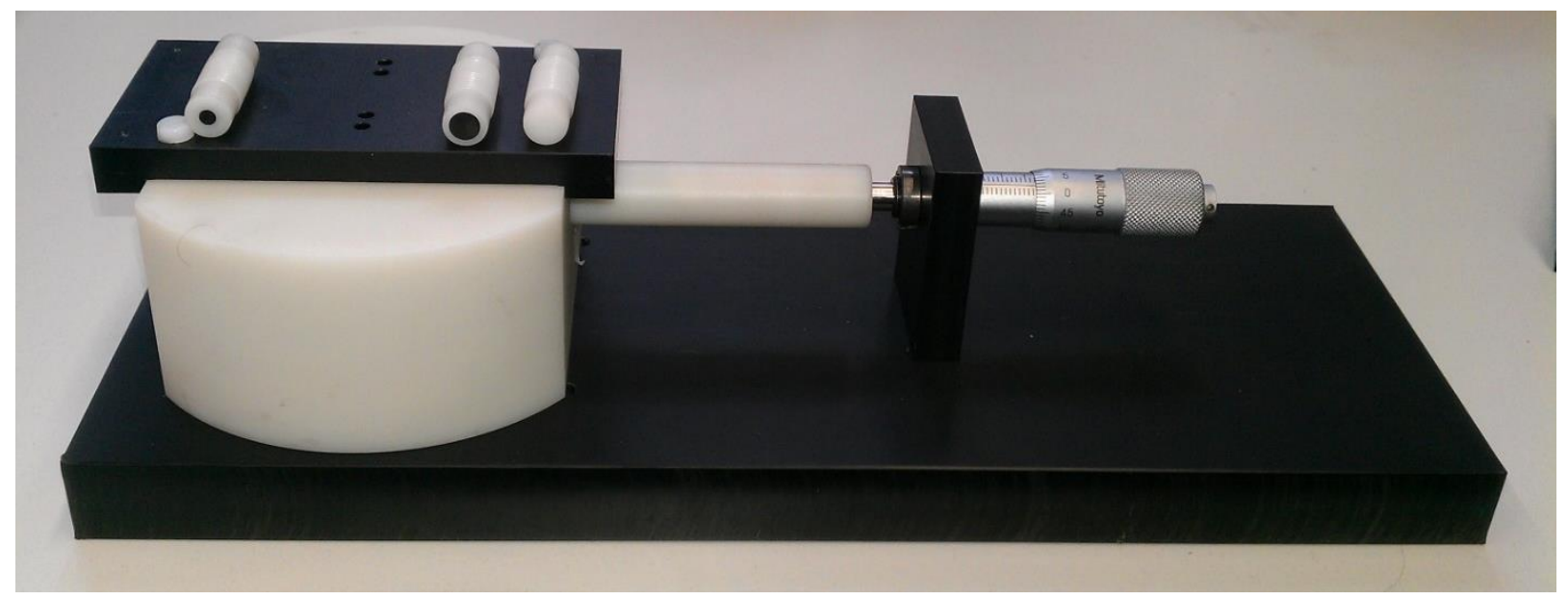

Figure 1. Photo of the solid phantom and of the cylinders embedding the black objects. 

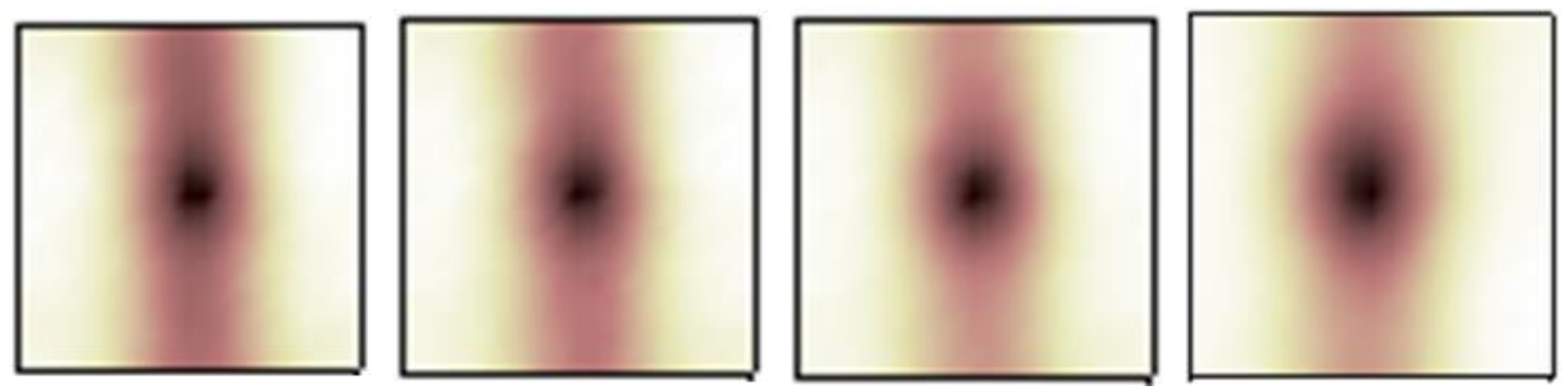

Figure 2. Normalized time-gated transmittance images of a $4 x 4 \mathrm{~cm}^{2}$ area around the inhomogeneity. The holding cylinder produces a shadow, yet the embedded black PVC cylinders are visible for all sizes (height=diameter=3, 4, 5, $7 \mathrm{~mm}$, from left to right).

\section{CONCLUSION}

In conclusion, we have proposed and constructed a solid phantom suitable to simulate realistic absorption changes within a diffusive medium using rods made of the same phantom matrix which embed small black objects. Although the rod itself - nominally identical to the phantom matrix - creates an optical perturbation, nevertheless, the effects of the small black objects are clearly detectable. Also, both X-scans and Z-scans can be easily produced by translating the rod, and taking the homogeneous region of the rod, far from the inclusion as reference state. The great value of the proposed phantom is that it is easily operated in a clinical environment, it is stable, rugged, and fairly reproducible, and can reproduce dynamic changes with no need to detach the fibers. Thus, it could be used for routine quality tests both of clinical instruments and of laboratory settings, and even be adopted in standards.
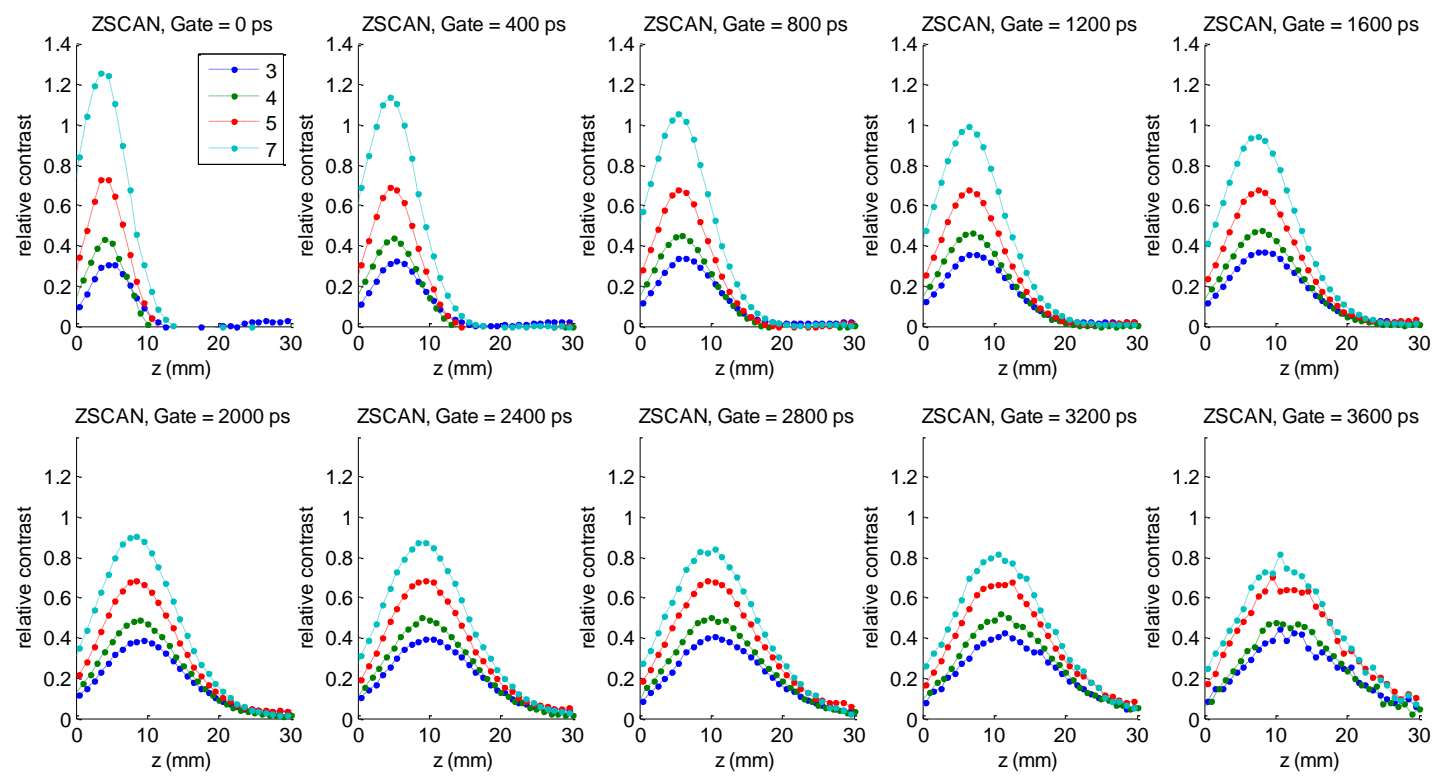

ZSCAN, Gate $=3600$ ps

Figure 3. Relative contrast derived from time-resolved reflectance measurements for different depths of the inclusion (Z-scan). The fibers are placed onto the lateral surface (on the left of Fig. 1).

\section{ACKNOWLEDGEMENTS}

The research leading to these results has partially received funding from the European Commission under the projects LASERLAB-EUROPE (n. 284464), OILTEBIA (n. 317526) and BabyLux (n. 620996). 


\section{REFERENCES}

[1] J. Hwang, J. Ramella-Roman, and R. Nordstrom, "Introduction: Feature issue on phantoms for the performance evaluation and validation of optical medical imaging devices," Biomed. Opt. Expr. 3, 1399-403 (2012).

[2] A. Torricelli, V. Quaresima, A. Pifferi, G. Biscotti, L. Spinelli, P. Taroni, M. Ferrari, R. Cubeddu, "Mapping of calf muscle oxygenation and haemoglobin content during dynamic plantar flexion exercise by multi-channel time-resolved near-infrared spectroscopy," Phys. Med. Biol. 49, 685-699 (2004).

[3] F. Martelli, A. Pifferi, D. Contini, L. Spinelli, A. Torricelli, H. Wabnitz, R. Macdonald, A. Sassaroli, and G. Zaccanti, "Phantoms for diffuse optical imaging based on totally absorbing objects. Part 1: basic concepts," $J$. Biomed. Opt. 18, 066014 (2013).

[4] F. Martelli, P. Di Ninni, G. Zaccanti, D. Contini, L. Spinelli, A. Torricelli, R. Cubeddu, H. Wabnitz, M. Mazurenka, R. Macdonald, A. Sassaroli, and A. Pifferi, "Phantoms for diffuse optical imaging based on totally absorbing objects, part 2: experimental implementation," J. Biomed. Opt. 19, 076011 (2014).

[5] L. Spinelli, M. Botwicz, N. Zolek, M. Kacprzak, D. Milej, P. Sawosz, A. Liebert, U. Weigel, T. Durduran, F. Foschum, A. Kienle, F. Baribeau, S. Leclair, J.-P. Bouchard, I. Noiseux, P. Gallant, O. Mermut, A. Farina, A. Pifferi, A. Torricelli, R. Cubeddu, H.-C. Ho, M. Mazurenka, H. Wabnitz, et al. "Determination of reference values for optical properties of liquid phantoms based on Intralipid and India ink," Biom. Opt. Expr. 5, $2037-$ 2053 (2014).

[6] H. Wabnitz, A. Jelzow, M. Mazurenka, O. Steinkellner, R. Macdonald, D. Milej, N. Zolek, M. Kacprzak, P. Sawosz, R. Maniewski, A. Liebert, S. Magazov, J. Hebden, F. Martelli, P. Di Ninni, G. Zaccanti, A. Torricelli, D. Contini, R. Re, L. Zucchelli, L. Spinelli, R. Cubeddu, and A. Pifferi, "Performance assessment of timedomain optical brain imagers, part 2: nEUROPt protocol," J. Biomed. Opt. 19, 086012 (2014).

[7] A. Pifferi, A. Torricelli, P. Taroni, D. Comelli, A. Bassi, R. Cubeddu, "Fully automated time domain spectrometer for the absorption and scattering characterization of diffusive media," Rev. Sci. Instrum. 78, 053103 (2007).

[8] A. Pifferi, A. Torricelli, L. Spinelli, D. Contini, R. Cubeddu, F. Martelli, G. Zaccanti, A. Tosi, A. Dalla Mora, F. Zappa, S. Cova, "Time-resolved diffuse reflectance using small source-detector separation and fast singlephoton gating," Phys. Rev. Lett. 100, 138101 (2008).

[9] A. Tosi, A. Dalla Mora, F. Zappa, A. Gulinatti, D. Contini, A. Pifferi, L. Spinelli, A. Torricelli, R. Cubeddu, "Fast-gated single-photon counting technique widens dynamic range and speeds up acquisition time in timeresolved measurements," Opt. Expr. 19, 10735-10746 (2011).

[10] A. Pifferi, A. Torricelli, R. Cubeddu, G. Quarto, R. Re, S. Konugolu, L. Spinelli, A. Farina, F. Martelli, H. Wabnitz, "Mechanically switchable solid inhomogeneous phantom for performance tests in diffuse imaging and spectroscopy," J. Biomed. Opt., in press (2015).

[11]P. Taroni, A., Pifferi, E., Salvagnini, L., Spinelli, A., Torricelli, R. Cubeddu, and A. Torricelli, "Sevenwavelength time-resolved optical mammography extending beyond $1000 \mathrm{~nm}$ for breast collagen quantification," Opt. Expr. 17, 15932-15946 (2009).

[12]R. Re, D. Contini, M. Turola, L. Spinelli, L. Zucchelli, M. Caffini, R. Cubeddu, and A. Torricelli, "Multichannel medical device for time domain "Multi-channel medical device for time domain functional near infrared spectroscopy based on wavelength space multiplexing," Biomed. Opt. Expr. 4, 2231-46 (2013). 